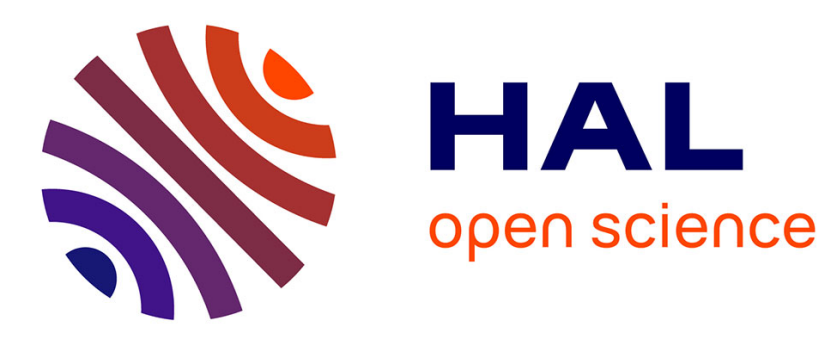

\title{
Radar differential reflectivity ZDR : a real-case evaluation of errors induced by antenna characteristics
}

Yves Pointin, Daniel Ramond, Jacques Fournet-Fayard

\section{To cite this version:}

Yves Pointin, Daniel Ramond, Jacques Fournet-Fayard. Radar differential reflectivity ZDR: a realcase evaluation of errors induced by antenna characteristics. Journal of Atmospheric and Oceanic Technology, 1988, pp.416-423. hal-01984656

\section{HAL Id: hal-01984656 \\ https://hal.uca.fr/hal-01984656}

Submitted on 3 Dec 2021

HAL is a multi-disciplinary open access archive for the deposit and dissemination of scientific research documents, whether they are published or not. The documents may come from teaching and research institutions in France or abroad, or from public or private research centers.
L'archive ouverte pluridisciplinaire HAL, est destinée au dépôt et à la diffusion de documents scientifiques de niveau recherche, publiés ou non, émanant des établissements d'enseignement et de recherche français ou étrangers, des laboratoires publics ou privés.

$$
\text { Copyright }
$$




\title{
Radar Differential Reflectivity $\boldsymbol{Z}_{\mathrm{DR}}$ : A Real-Case Evaluation of Errors Induced by Antenna Characteristics
}

\author{
YVES POINTIN, DANEL RAMOND AND JACQUES FouRNET-FAYARD \\ L.A.M.P./O.P.G.C., Université de Clermont-Ferrand II, Aubière, France
}

(Manuscript received 29 May 1987, in final form 18 November 1987)

\section{ABSTRACT}

\begin{abstract}
Multiple parameter radar measurements (dual frequency hail signal DFR-HS or differential reflectivity $Z_{\mathrm{DR}}$ ) can be strongly affected by the mismatch of the antenna illumination functions at the two wavelengths or polarizations. The resulting instrumental error can be estimated, with some approximations, from the independently measured reflectivity at one wavelength or polarization by using the actual radar illumination functions. This objective estimation of the antenna-induced error is made in some real cases and its possible consequences on the data analysis are discussed.
\end{abstract}

\section{Introduction}

Multiple parameter radar measurements add a new parameter $Z_{\alpha \beta}$ to the usual cloud reflectivity $Z$, defined as the ratio of the reflectivity factors $\left(Z_{\alpha}\right.$ and $\left.Z_{\beta}\right)$ at two different wavelengths or polarizations $\alpha$ and $\beta$ (Johnson 1984). For example, the hail signal (Atlas and Ludlam $1961)$ is the ratio of the S-band (10 cm wavelength) reflectivity factor $Z_{10}$ to the attenuation corrected $X$ band ( $3 \mathrm{~cm}$ wavelength) reflectivity factor $Z_{3}$. Similarly, the differential reflectivity $Z_{D R}$ (Seliga and Bringi 1976) is the ratio of the reflectivity factor $Z_{h}$ in the horizontal polarization to the reflectivity factor $Z_{v}$ in the vertical polarization.

This ratio parameter $Z_{\alpha \beta}$ has a larger noise-over-signal ratio than any of the reflectivity factors and is more affected by instrumental errors. In particular, each reflectivity factor $Z_{\alpha}$ depends upon the feed horn and the aerial characteristics for the corresponding wavelength or polarization $\alpha$. For single parameter measurements, the measurement errors due to the beam pattern are well known (Donaldson 1964, 1965; Zawadski 1982). For multiple parameter measurements, these errors have recently been studied by Rinehart and Tuttle (1982, 1984a), Jameson and Heymsfield (1984), Johnson (1984), Herzegh and Carbone (1984) and by Herzegh and Conway (1986). These studies have shown that the main source of error is the mismatch of the illumination function of the antenna at the two wavelengths or polarizations $\alpha$ and $\beta$.

Rinehart and Tuttle (1982) have estimated these measurement errors for the hail signal in some real

Corresponding author address: Dr. Y. Pointin, Université de Clermont II, LAMP/OPGC, UA/CNRS No. 267, 63170 Aubiere, France. cases, and Herzegh and Carbone (1984) have estimated the differential reflectivity errors in some simple cases. These two studies have used the actual illumination functions of the National Center for Atmospheric Research (NCAR) CP-2 radar (Keeler et al. 1984).

In this study, we use the same kind of measurements for the OPGC $10 \mathrm{~cm}$ ANATOL radar in a three-dimensional numerical simulation model in order to estimate the differential reflectivity errors, induced by the feed horn and by the aerial in some real cases. As pointed out by Jameson and Heymsfield (1984) and by Rinehart and Tuttle (1984b), these estimated errors can indicate the zones where the measured values are strongly contaminated and the zones where the measured values can be used with confidence. These estimated errors cannot be used to correct the contaminated values because of the uncertainties of this estimation which is based on contaminated measured values and which does not take into account the ground echo effects. Therefore, these estimated errors are useful to detect anomalous differential reflectivity values and to prevent erroneous analysis of the data.

The formula used for the estimation of the ratio parameter error $\Delta Z_{\alpha \beta}$ is derived in section 2 . The practical steps which must be followed during this error evaluation are described in the third section. The ANATOL radar characteristics are given in the fourth section and the estimated errors computed in real cases are discussed in the fifth section.

\section{Ratio parameter error $\Delta Z_{\mathrm{DR}}$}

The reflectivity factor $\bar{Z}_{\alpha}$ measured by the radar for any given wavelength or polarization $\alpha$, and at the elevation $\theta$, azimuth $\phi$ and radial distance $r$ from the radar is, in fact, the averaged value of the actual cloud re- 
flectivity field $Z_{\alpha}$ in the $r$-sphere around the radar. This averaged value is weighted by the illumination function of the antenna (Donaldson 1965; Zawadski 1982; Johnson 1984):

$$
\bar{Z}_{\alpha}(r, \theta, \phi)=C_{\alpha} \int_{\Omega^{\prime}} f_{\alpha}{ }^{2}\left(\gamma^{\prime}, \psi^{\prime}\right) Z_{\alpha}\left(x^{\prime}, y^{\prime}, z^{\prime}\right) d \Omega^{\prime}
$$

where $\Omega^{\prime}$ is the unit sphere centered on the antenna focus; $x^{\prime}, y^{\prime}$ and $z^{\prime}$ are the Cartesian coordinates of any point $M^{\prime}$ situated at a distance $r$ from the antenna (see Fig. 1 for the geometrical setting):

$$
x^{\prime 2}+y^{\prime 2}+z^{\prime 2}=r^{2}
$$

$\gamma^{\prime}$ and $\psi^{\prime}$ are the polar angles of $M^{\prime}$, relative to the beam axis; and $f_{\alpha}$ is the one-way power illumination function of the antenna. In deriving (1), emission and reception are assumed to be made by the same antenna at the same wavelength or polarization $\alpha$. Generalizations to other cases are straightforward. The normalization constant $C_{\alpha}$ in (1) is the reciprocal of the squared illumination function integral over the unit sphere.

The illumination function $f_{\alpha}$ depends upon the antenna characteristics which induce an irregular sidelobe distribution. This function can be split into an ideal part $\delta$, which is the axially symmetrical Gaussian fit to the main lobe, and a small perturbation part as follows:

$$
f_{\alpha}^{2}\left(\gamma^{\prime}, \psi^{\prime}\right)=\delta\left(\gamma^{\prime}\right)+\epsilon g_{\alpha}\left(\gamma^{\prime}, \psi^{\prime}\right)
$$

where $\epsilon$ is a small parameter which can be used to expand any function into a power series. The ideal part is assumed to be independent of the wavelength or polarization $\alpha$ (same beamwidth for the two wavelengths

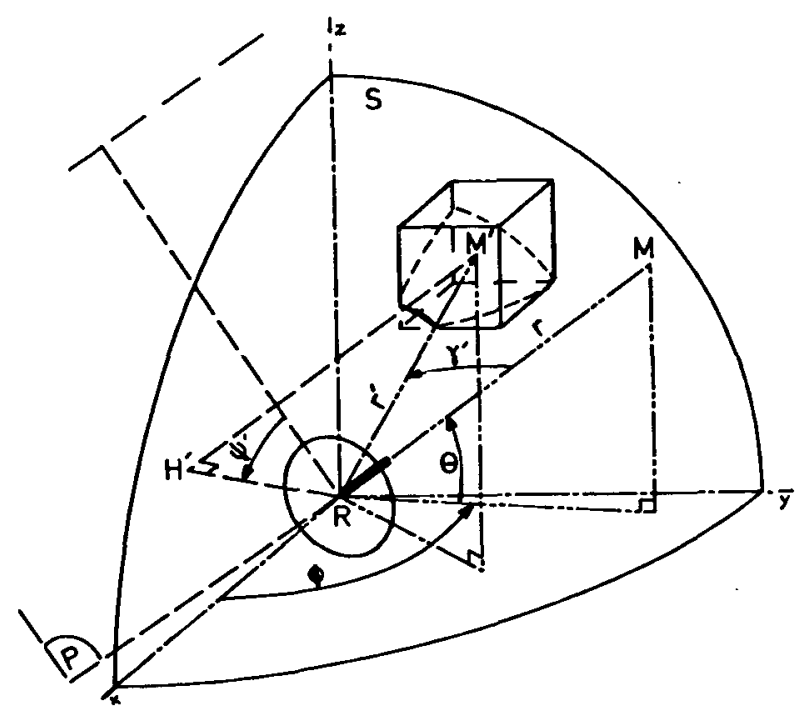

FIG. 1. Geometrical sketch of the radar antenna $R$ pointing toward the $\mathrm{M}$ point, of polar angles $\theta$ and $\phi$. The $r$-sphere $S$, centered at the radar antenna and passing through $M$, intersects the grid mesh centered at the $\mathrm{M}^{\prime}$ point. The projection plane $\mathrm{P}$ is perpendicular to the beam axis $R M$ and includes the $\mathrm{M}^{\prime}$-projection point called $\mathrm{H}^{\prime}$. The antenna relative polar angles of $\mathbf{M}^{\prime}$ are $\gamma^{\prime}$ and $\psi^{\prime}$. or polarizations). The perturbation part $g_{\alpha}$ induces only small errors in single parameter measurements except where large cloud reflectivity gradients are found (Donaldson 1964; Zawadski 1982).

In multiple parameter measurements, the ratio parameter $\bar{Z}_{\alpha \beta}$ is defined from the ratio of the measured reflectivity $\bar{Z}_{\alpha}$ at the wavelength or polarization $\alpha$ to that $\bar{Z}_{\beta}$ at the wavelength or polarization $\beta$ :

$$
\bar{Z}_{\alpha \beta}=10 \log \left(\bar{Z}_{\alpha} / \bar{Z}_{\beta}\right) \text {. }
$$

This ratio parameter can be expanded up to the first power in $\epsilon$ as:

$$
\begin{array}{r}
\bar{Z}_{\alpha \beta}(r, \theta, \phi)=10 \log \left(\int_{\Omega^{\prime}} \delta Z_{\alpha} d \Omega^{\prime} / \int_{\Omega^{\prime}} \delta Z_{\beta} d \Omega^{\prime}\right) \\
+10 \log \left(C_{\alpha} / C_{\beta}\right)+10 \log \left[1+\epsilon\left(\int_{\Omega^{\prime}} g_{\alpha} Z_{\alpha} d \Omega^{\prime}\right)\right. \\
\left.\left.\int_{\Omega^{\prime}} \delta Z_{\alpha} d \Omega^{\prime}-\int_{\Omega^{\prime}} g_{\beta} Z_{\beta} d \Omega^{\prime} / \int_{\Omega^{\prime}} \delta Z_{\beta} d \Omega^{\prime}\right)\right]
\end{array}
$$

where the variable dependence of functions and fields has not been written inside the integrals. The first term on the right-hand side of (4) is the ideal ratio parameter defined from the ratio of the cloud reflectivity averages within the radar main lobe. This ideal ratio parameter provides an unbiased estimate of the mean cloud reflectivity ratio (Bringi et al. 1983; Sachidananda and Zrnić 1985). The second term is a constant which can be deduced from a calibration procedure (for example, rotation of the vertically pointing antenna in light rain without strong wind). The third term on the right-hand side of (4) is the ratio parameter error $\Delta Z_{\alpha \beta}$ which is due to the deviation of the illumination functions from their ideal form and which depends on the cloud reflectivity fields over the $r$-sphere centered at the antenna focus.

In order to be able to compute an estimation of this ratio parameter error $\Delta Z_{\alpha \beta}$, some approximations must be done which should not change the order of magnitude of this error. At first, the values of the reflectivity $Z_{\alpha}$ are used instead of $Z_{\beta}$ in both integrals in the third term within the third logarithm on the righthand side of (4). This approximation is valid when the relative variations of $Z_{\beta}$ are of the same order of magnitude as that of $Z_{\alpha}$ since this reflectivity factor only appears as a weighting function of the small illumination perturbation part $g_{\beta}$. Therefore, this approximation is valid when the cloud ratio parameter $Z_{\alpha \beta}$ is smaller than a few dB. This is almost always true for the differential reflectivity $Z_{\mathrm{DR}}$ which is usually less than $6 \mathrm{~dB}$. A second approximation is made by replacing the cloud reflectivity factor $Z_{\alpha}$ by the measured reflectivity factor $\bar{Z}_{\alpha}$ which is simultaneously recorded with the measured ratio parameter $\bar{Z}_{\alpha \beta}$. This approximation is only valid when the cloud reflectivity gradients are small within the resolution volume (Don- 
aldson 1965; Zawadski 1982) and when the measured reflectivity is not affected by ground clutter through sidelobe contamination. Indeed, in some cases, the cloud reflectivity gradients and maximum values may differ significantly from the corresponding measured ones and this second approximation may not hold. In these cases, however, the ratio parameter error based on the measured values, although smaller than the true error, is still likely to be large and to indicate that the differential reflectivity measurements are erroneous. With these two approximations, the ratio parameter error $\Delta Z_{\alpha \beta}$ can be computed from:

$$
\begin{aligned}
\Delta Z_{\alpha \beta}(r, \theta, \phi)=10 \log [1+ & C_{\alpha} \int_{\Omega^{\prime}}\left(f_{\alpha}^{2}-f_{\beta}^{2}\right) \\
& \left.\times \bar{Z}_{\alpha} d \Omega^{\prime} / \bar{Z}_{\alpha}(r, \theta, \phi)\right]
\end{aligned}
$$

where the one-way illumination functions $f_{\alpha}$ and $f_{\beta}$ at both wavelengths or polarizations $\alpha$ and $\beta$ have been reintroduced. Inside the integral in (5), these functions depend on the polar coordinates $\gamma^{\prime}$ and $\psi^{\prime}$ and the measured reflectivity factor $\bar{Z}_{\alpha}$ depends on the Cartesian coordinates $x^{\prime}, y^{\prime}$ and $z^{\prime}$ as shown in Fig. 1.

Equation (5) shows that the mismatch of the illumination functions at wavelengths or polarizations $\alpha$ and $\beta$ is the major source of the ratio parameter error $\Delta Z_{\alpha \beta}$. This mismatch, highly probable in the irregular sidelobes, may also occur in the main lobe, inducing large errors in this case because of the corresponding high values of the illumination functions.

This ratio parameter error $\Delta Z_{\alpha \beta}$ is also related to the measured reflectivity factor $\bar{Z}_{\alpha}$ and, for any radar, some peculiar reflectivity fields may induce significant errors in some small cloud parts (Donaldson 1965; Herzegh and Conway 1986). Therefore, it is important to estimate this ratio parameter error before reaching any conclusions from the measured ratio parameter.

\section{Practical steps of the $\Delta Z_{\alpha \beta}$ error evaluation from real data}

Equation (5) shows that the ratio parameter error $\Delta Z_{\alpha \beta}$ results from the convolution product, over the unit sphere, of the illumination difference function $\left(f_{\alpha}^{2}\right.$ $-f_{\beta}^{2}$ ) by the measured reflectivity factor $\bar{Z}_{\alpha}$ over the $r$-sphere centered at the antenna focus (Fig. 1). Therefore the illumination functions of the antenna must be measured at both wavelengths or polarizations $\alpha$ and $\beta$, as done by Keeler et al. (1984). The measured values are then interpolated for all polar angles $\gamma^{\prime}$ and $\psi^{\prime}$ by Fourier series or any other interpolating method.

Then, for each real case the measured reflectivity field must be known at a given reference time $t_{0}$ for all Cartesian coordinates $(x, y, z)$. This is simply done by interpolating the radar data measured during a volume scan, at all the points $M$ of a three-dimensional regular rectangular grid in which the analyzed cloud is included. This interpolation must be done at the reference time $t_{0}$, by taking into account the horizontal motion of the cloud during the volume scan.

The ratio parameter error $\Delta Z_{\alpha \beta}$ is evaluated at all grid points $M$ by approximating the convolution integral in (5) by the sum over all rectangular mesh (at $M^{\prime}$ ), which are cut out by the $r$-sphere centered at the radar and passing by $M$ (Fig. 1). From the Cartesian coordinates, $x, y, z$ of $M$ and $x^{\prime}, y^{\prime}, z^{\prime}$ of $M^{\prime}$, the antenna relative polar angles $\gamma^{\prime}$ and $\psi^{\prime}$ can be easily computed and the values of the illumination functions appearing in (5) are deduced from the measured values. The unit sphere differential area $d \Omega^{\prime}$ is computed from the normalized area of the part of the $r$-sphere inside the $M^{\prime}$ rectangular mesh, as shown in Fig. 1.

The calculated convolution integral is then normalized by the $C_{\alpha}$ constant and by the measured value of the reflectivity factor $\bar{Z}_{\alpha}$ at the grid point $M$ and the ratio parameter $\Delta Z_{\alpha \beta}$ is estimated from (5). These computations are straightforward but lengthy and may require a large amount of computer time.

\section{The ANATOL radar characteristics}

The $10 \mathrm{~cm}$ ANATOL radar (Cordesses et al. 1983) uses a $4 \mathrm{~m}$ parabolic aerial formed by two rigid half parts bolted together and a Potter feed horn for the emission and reception of horizontally (index $h$ ) or vertically (index $v$ ) polarized electromagnetic waves.

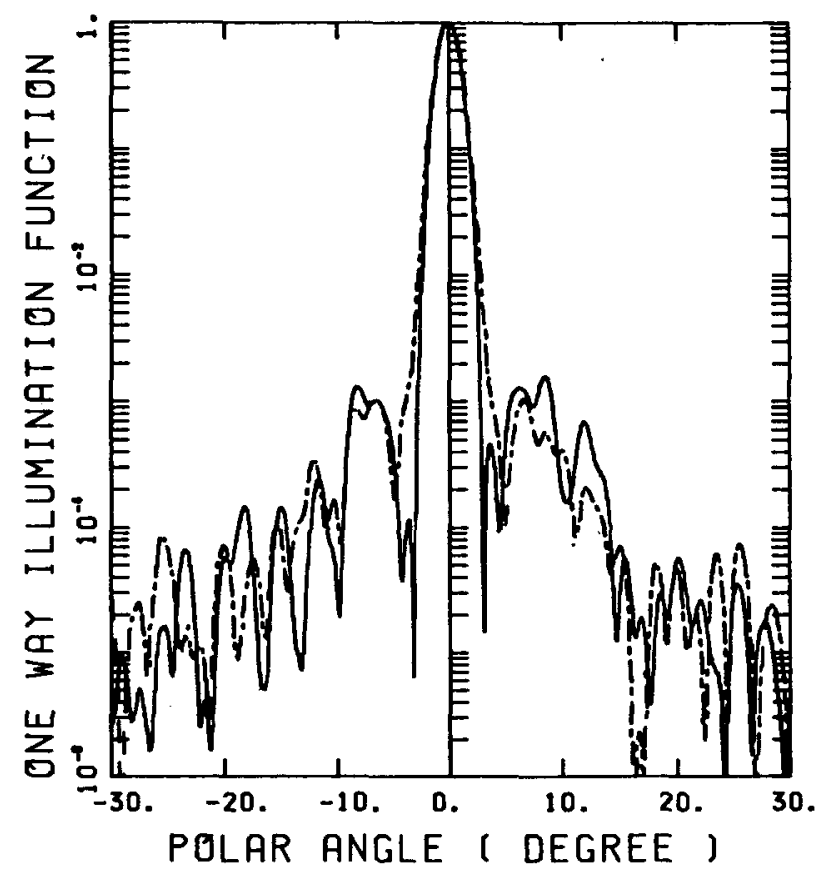

Fig. 2. One-way illumination functions in the horizontal polarization $\left(f_{h}\right.$ in full line) and in the vertical polarization $\left(f_{v}\right.$ in dashdotted line) vs the antenna relative polar angle $\gamma^{\prime}$ in degrees for the horizontal plane $\left(\psi^{\prime}=0\right.$ and $\left.\psi^{\prime}=\pi\right)$. 
The radar polarization is switched from horizontal to vertical on a pulse-to-pulse basis by a high power ferrite circulator every $2 \mathrm{~ms}$. The return powers in the horizontal and in the vertical polarizations go through a logarithmic amplifier before being averaged over 64 of their respective pulses and further averaged over four consecutive subgates. Therefore, every $0.256 \mathrm{~s}$ the mean power at the horizontal polarization and the ratio of the mean power of horizontal to vertical polarizations are recorded in up to 1024 gates of $120 \mathrm{~m}$ width, together with the time, the elevation and the azimuth angles of the last averaging pulse. Usually, the antenna rotates at a rate of $8 \mathrm{deg} \mathrm{s}^{-1}$ for which the averaging angle is comparable to the half-power beamwidth of $1.8 \mathrm{deg}$ of the antenna.

The illumination functions $f_{h}$ and $f_{v}$ at both polarizations of the Potter feed horn and aerial ensemble have been measured on a test bench of the CNET (Behe and Nurdin 1984) with a 0.1 degree resolution along the horizontal $\left(\psi^{\prime}=0\right.$ and $\left.\pi\right)$ and along the vertical $\left(\psi^{\prime}=\pi / 2\right.$ and $\left.3 \pi / 2\right)$ planes. These one-way illumina- tion functions are drawn in Fig. 2 vs the antenna polar angle $\gamma^{\prime}$ along the horizontal plane. These normalized functions are below $-28 \mathrm{~dB}$ in the sidelobes $(-22 \mathrm{~dB}$ along the vertical plane not shown), but the illumination function in the vertical polarization is larger than that in the horizontal polarization within the main lobe.

These measured values are interpolated for all relevant polar angles $\gamma^{\prime}$ and $\psi^{\prime}$ (up to $30 \mathrm{deg}$ ) by a linear interpolation in $\gamma^{\prime}$ and by a four-term Fourier series in the azimuthal angle $\psi^{\prime}$. The interpolated difference $f_{h}^{2}-f_{v}^{2}$ is drawn in the center part of the $\gamma^{\prime}-\psi^{\prime}$ plane in Fig. 3. For the ANATOL antenna, the absolute difference is only significant within $3 \mathrm{deg}$ of the beam axis, i.e., in the main lobe, and it reaches its maximum value of 0.4 within $1 \mathrm{deg}$ of the beam axis. Within the main lobe, this difference is mainly negative with some small positive values along the horizontal plane. However, values outside the half-power beamwidth zone may contribute significantly to the convolution integral in Eq. (5) since they are weighted by the measured

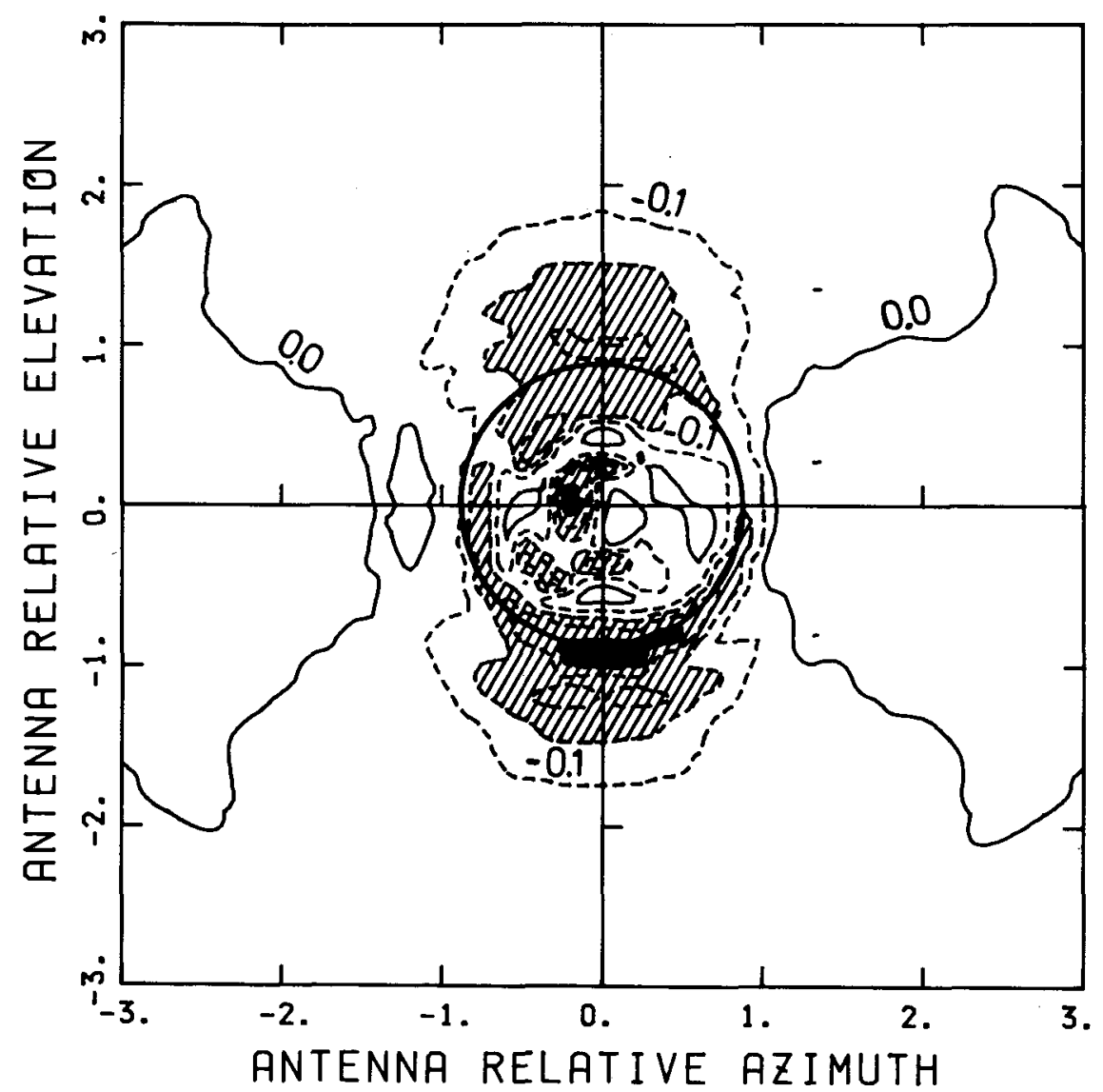

FIG. 3. Isolines of the illumination difference function $\left(f_{h}^{2}-f_{v}{ }^{2}\right)$ in the antenna-relative azimuth and elevation angles (in degrees). These angles are related to the antenna-relative polar angles ( $\gamma^{\prime}$ and $\psi^{\prime}$ ) defined in Fig. 1. Isolines are drawn every 0.1 unit (dimensionless) and dashed isolines correspond to negative values. The hatched areas correspond to values below -0.2 and the dark areas to values below -0.4 . The half-power beamwidth is indicated by the thick line circle. 
reflectivity $\bar{Z}_{h}$ which may change its order of magnitude in short distances.

\section{Differential reflectivity errors in real cases}

These errors have been computed in several real cases but only one example will be discussed below, since the consequences of these errors appear to be the most important in this case.

\section{a. Experimental conditions}

On 4 October, 1985 some isolated convective clouds developed in a general southeast circulation at all levels. The surface temperature was near $10^{\circ} \mathrm{C}$ and the $0^{\circ} \mathrm{C}$ isotherm was $2 \mathrm{~km}$ above the radar. A developing cloud passed above the radar with estimated velocities of 12 $\mathrm{m} \mathrm{s}^{-1}$ toward the east and $5 \mathrm{~m} \mathrm{~s}^{-1}$ toward the north. These velocities are assumed to be independent of altitude, position and time, and are deduced from the comparison of two similar PPI or CAPPI scans obtained at low level before and after the reference time $t_{0}$. The volume scan is obtained by successive sweeps at constant elevation angle of a $100 \mathrm{deg}$ sectorial area. At the end of each sweep, the elevation angle increases by around $1.8 \mathrm{deg}$, rising from 0 to $21 \mathrm{deg}$, and then decreases back to zero in $1.8 \mathrm{deg}$ steps. This volume scan takes about 4 min to be completed.

The size of the interpolating grid is $0.12 \mathrm{~km}$ horizontally and $0.5 \mathrm{~km}$ vertically, and the mesh covers a $30 \times 30 \mathrm{~km}$ area situated in the northeast quadrant of the radar, between the altitudes of 1.0 and $8.5 \mathrm{~km}$ above the radar. With a maximum elevation angle of $21 \mathrm{deg}$, some measurement data are lacking at high altitudes and at short distances from the radar. This, among other reasons, implies that the convolution integral appearing in Eq. (5) is underestimated because the $r$ sphere, on which it is evaluated, includes large areas where the cloud reflectivity may be significant but there is no measured data.

\section{b. Data description}

A constant altitude PPI (CAPPI), made at $1.5 \mathrm{~km}$ above the radar and at the reference time $t_{0}=1601$, is shown in Fig. 4. At this time, the cloud is located between 15 and $20 \mathrm{~km}$ from the radar and the maximum horizontal reflectivity $\bar{Z}_{h}$ is above $50 \mathrm{dBZ}$ in this horizontal cross section. A vertical cross section of the horizontal reflectivity, made along the $A-B$ axis in Fig. 4 , is shown in Fig. 5. The $40 \mathrm{dBZ}$ isocontour reaches the altitude of $5 \mathrm{~km}$ while the cloud top is well above

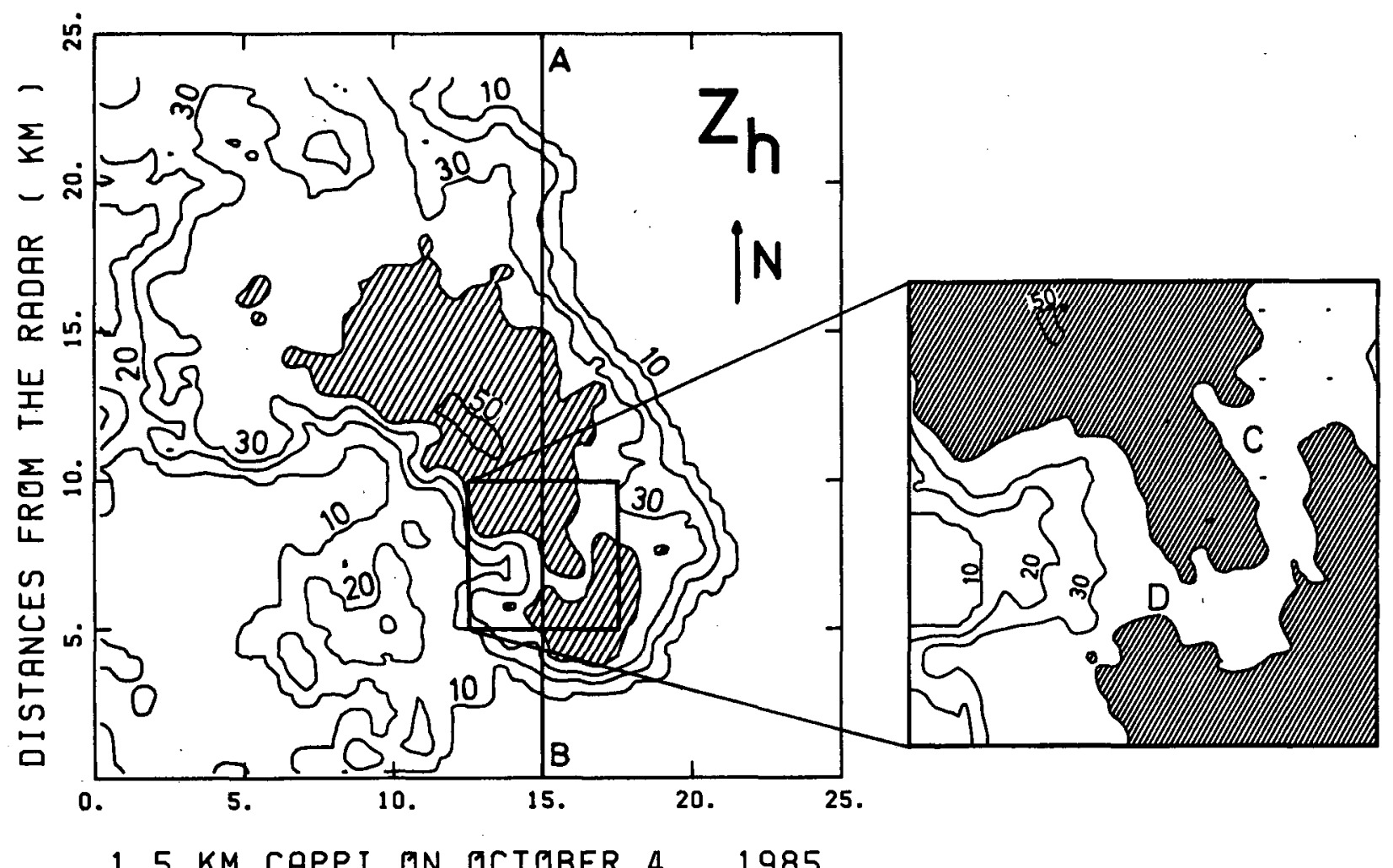

FIG. 4. Horizontal cross section made at the $1.5 \mathrm{~km}$ altitude (CAPPI) on 4 October 1985 of the measured horizontal reflectivity $\bar{Z}_{h}$. Isolines are drawn every $10 \mathrm{dBZ}$ starting from $10 \mathrm{dBZ}$. The zones where $\bar{Z}_{h}$ exceeds $40 \mathrm{dBZ}$ are hatched. The inset shows the detailed features of the reflectivity field in the indicated square area. 


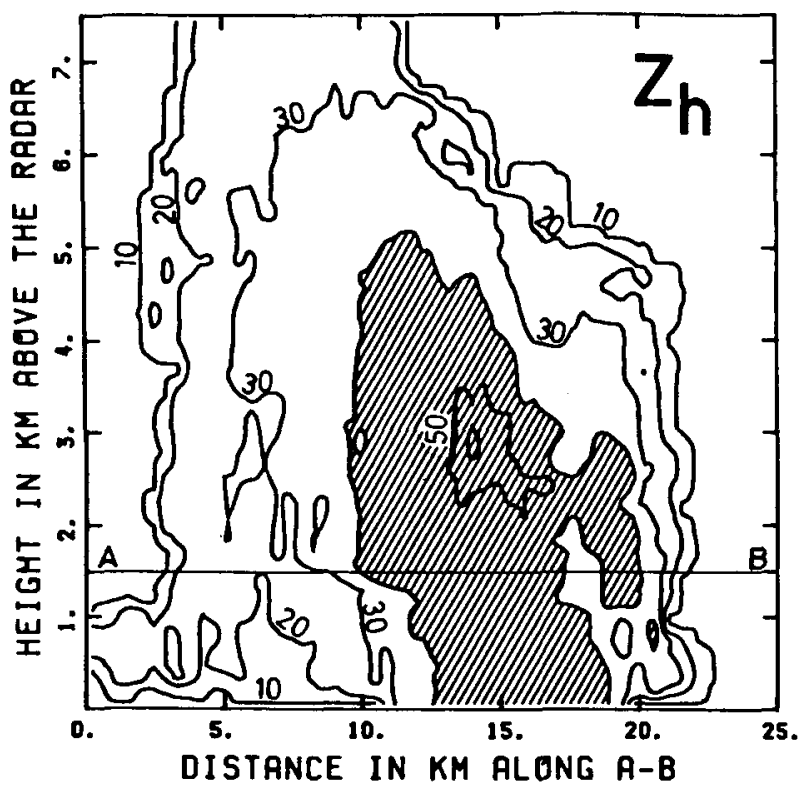

FIG. 5. Vertical cross section of the measured horizontal reflectivity $\bar{Z}_{h}$ reconstructed along the $A-B$ axis of Fig. 4 from the volume scan. Isolines are drawn every $10 \mathrm{dBZ}$ starting from $10 \mathrm{dBZ}$. The zones where $\bar{Z}_{h}$ exceeds $40 \mathrm{dBZ}$ are hatched.

$7.5 \mathrm{~km}$. The maximum horizontal reflectivity is also higher than $50 \mathrm{dBZ}$ in this vertical cross section and appears between the altitudes of 2.5 and $3.5 \mathrm{~km}$. Fur- ther analyses show that the $50 \mathrm{dBZ}$ reflectivity core takes the form of a hook region rising above the 50 $\mathrm{dBZ}$ isoline in Fig. 4 and with an overhang part (in the southeast direction) which appears in the vertical cross section in Fig. 5 at a distance of $15 \mathrm{~km}$ and above $2 \mathrm{~km}$. On the southeast part of this cloud $(x=16 \mathrm{~km}$, $y=4 \mathrm{~km}$ in Fig. 4 and $x^{\prime}=20 \mathrm{~km}$ in Fig. 5) a separated cell appears in a developing stage. The details of the reflectivity pattern of the separation zone between these two cells are shown by the inset in Fig. 4.

\section{c. Differential reflectivity error $\Delta Z_{D R}$}

The differential reflectivity error $\Delta Z_{\mathrm{DR}}$ is computed from the three-dimensional reflectivity field described above, following the procedure developed in section 3 . The same $1.5 \mathrm{~km}$ horizontal cross section as in Fig. 4 is shown in Fig. 6 for this differential reflectivity error $\Delta Z_{\mathrm{DR}}$. In this last figure, the hatched areas correspond to the areas where the measured horizontal reflectivity exceeds $40 \mathrm{dBZ}$, as shown in Fig. 4. The differential reflectivity error $\Delta Z_{\mathrm{DR}}$ is mainly negative, with minimum and maximum values of -0.8 and $+0.6 \mathrm{~dB}$, respectively. As expected (Johnson 1984; Herzegh and Carbone 1984; Herzegh and Conway 1986), the largest values of $\Delta Z_{\mathrm{DR}}$ appear on the edge of the $40 \mathrm{dBZ}$ areas, particularly in the neighborhood of the $C$ and $D$ points, in the separation zone between the two cells, characterized by large horizontal reflectivity gradients. How-

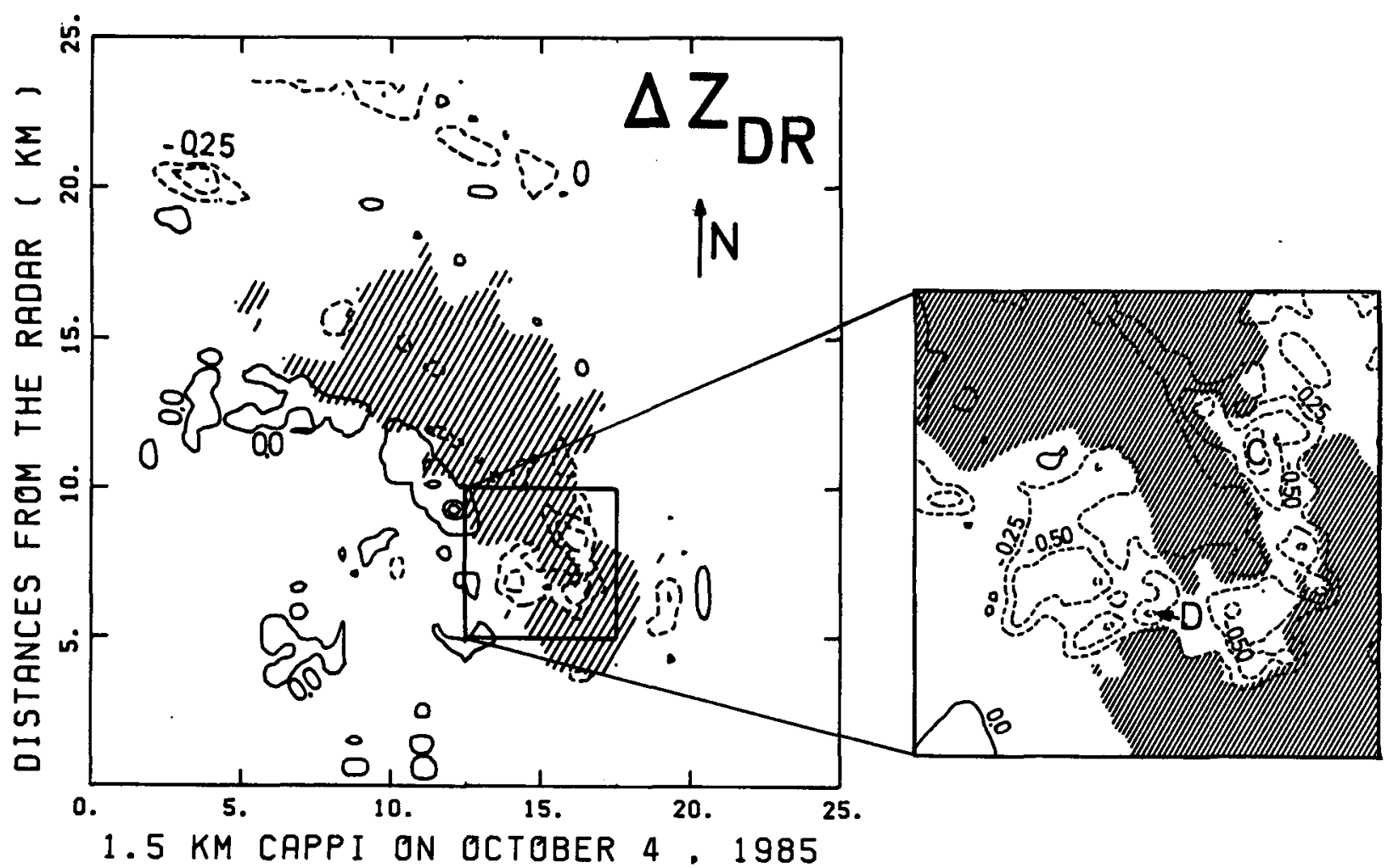

FIG. 6. As in Fig. 4 except for the differential reflectivity error $\Delta Z_{\mathrm{DR}}$. Isolines are drawn every $0.25 \mathrm{~dB}$ and dashed isolines correspond to negative values. The hatched zones are zones where the measured horizontal reflectivity $\bar{Z}_{h}$ exceeds $40 \mathrm{dBZ}$ as shown in Fig. 4 . 
ever, in this case, some large values of $\Delta Z_{\mathrm{DR}}$ also appear inside the $40 \mathrm{dBZ}$ areas in a somewhat more uniform region at the top of the inset in Fig. 6. This feature may result from the existence of the hook echo region which overhangs the $40 \mathrm{dBZ}$ areas in this $1.5 \mathrm{~km}$ horizontal cross section (as shown in Fig. 5 for a distance of $15 \mathrm{~km}$ from $A$ ) leading to large vertical reflectivity gradients there.

The measured values of the differential reflectivity $\bar{Z}_{\mathrm{DR}}$ are shown in Fig. 7 for the same $1.5 \mathrm{~km}$ horizontal cross section. In this figure, the hatched areas also correspond to the areas where the horizontal reflectivity exceeds $40 \mathrm{dBZ}$, as shown in Fig. 4. Figure 7 shows that the measured differential reflectivity $\bar{Z}_{D R}$ is close to $3 \mathrm{~dB}$ inside the $40 \mathrm{dBZ}$ hatched areas, reaches $4 \mathrm{~dB}$ inside the southeast developing part of the cloud and takes minimum values close to $2 \mathrm{~dB}$ in the northwest part.

The inset in Fig. 7 shows that the zone in which the measured differential reflectivity exceeds $4 \mathrm{~dB}$ is made of two separated parts situated west and east of the $C$ point, respectively. The same inset in Fig. 6 indicates that the differential reflectivity error is large and negative just between these two separated $4 \mathrm{~dB}$ isolines. More generally, the measured differential reflectivity $\bar{Z}_{\mathrm{DR}}$ exhibits a dip in the neighborhood of the $C$ and $D$ points for which the differential reflectivity error $\Delta Z_{\mathrm{DR}}$ reaches its minimum values of $-0.8 \mathrm{~dB}$, as shown in the inset of Fig. 6. Therefore, this dip of the measured differential reflectivity may be partly induced by the antenna characteristics, and may not represent a true spatial variation of the microphysical properties of the cloud.

\section{Conclusions}

In multiple parameter radar measurements, a ratio parameter $Z_{\alpha \beta}$ is defined as the ratio of the measured reflectivity factor in two different wavelengths or polarizations $\alpha$ and $\beta$. The fact that the illumination functions of the antenna in each wavelength or polarization may be different in the sidelobes, but also in the main lobe, may induce instrumental errors in the measurements of this ratio parameter. This ratio parameter error can be estimated under two approximations; namely, replacing the vertical reflectivity factor by the horizontal one and using the measured horizontal reflectivity factor instead of the true cloud reflectivity factor. This estimation is given by the twodimensional convolution product, over the $r$-sphere centered at the antenna focus, of the reflectivity factor $\bar{Z}_{\alpha}$, independently measured at the wavelength or polarization $\alpha$, by the difference of the two-way illumination functions in each wavelength or polarization. This error estimation must be done from the three-

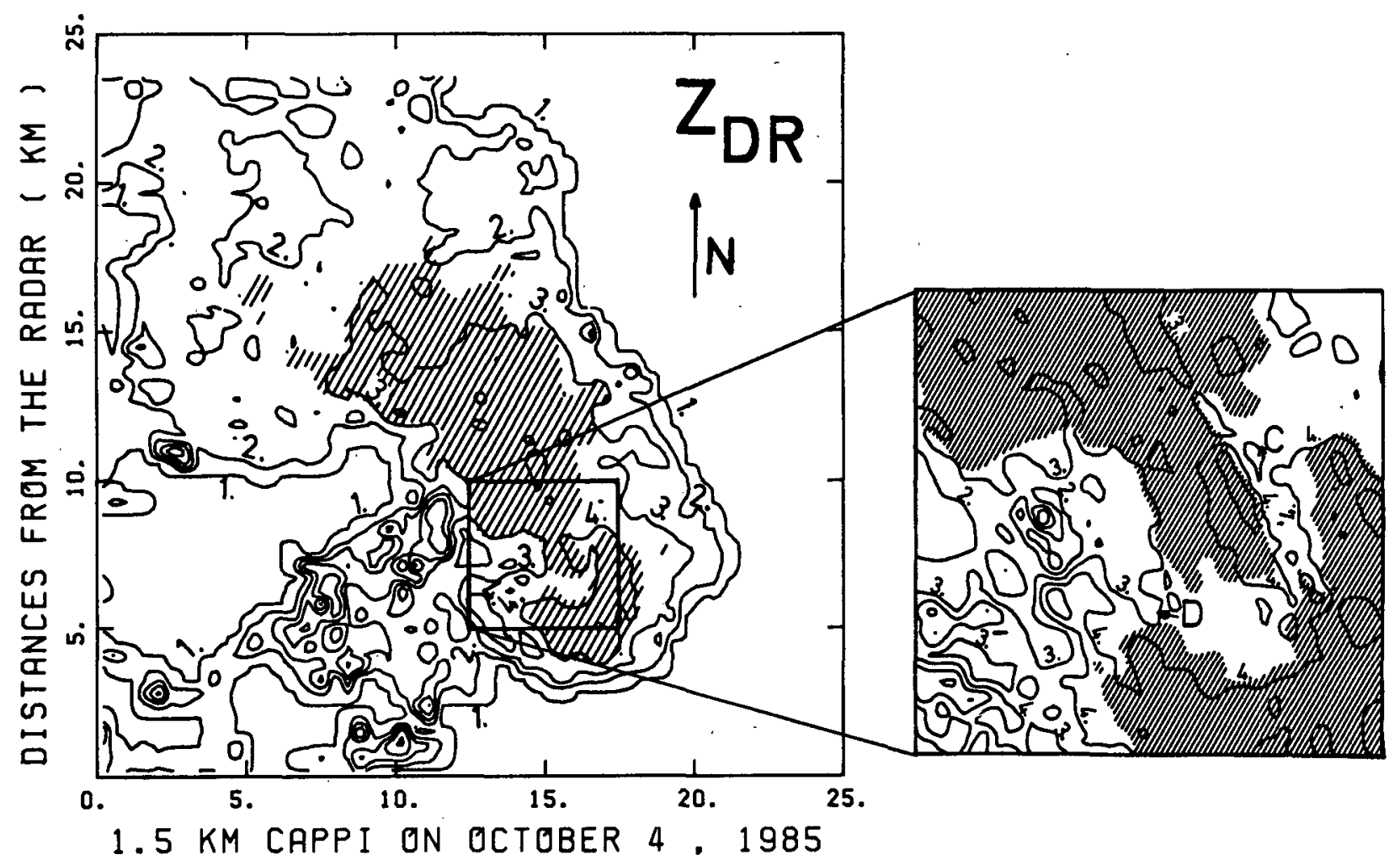

FIG. 7. As in Fig. 4 except for the measured differential reflectivity $\bar{Z}_{\mathrm{DR}}$. Isolines are drawn every $1 \mathrm{~dB}$ starting from $1 \mathrm{~dB}$. The hatched zones are zones where the measured horizontal reflectivity $\bar{Z}_{h}$ exceeds $40 \mathrm{dBZ}$ as shown in Fig. 4 . 
dimensional $Z_{\alpha}^{\prime}$ reflectivity field interpolated at a reference time $t_{0}$, from the data measured during a volume scan including the analyzed cloud.

The differential reflectivity errors have been estimated in several real cases, using the actual illumination functions of the $10 \mathrm{~cm}$ ANATOL radar. In general, the differential reflectivity error takes significant values (up to $0.8 \mathrm{~dB}$ for the ANATOL antenna) only in zones of large horizontal or vertical reflectivity gradients. Therefore, these zones appear on the edge of the horizontal reflectivity maxima in most horizontal or vertical cross sections and can easily be detected. In some cases, however, the horizontal reflectivity maximum can be situated outside of the analyzed cross section and can induce in it anomalous values of the differential reflectivity which cannot be detected. Therefore, the estimation of the differential reflectivity error $\Delta Z_{\mathrm{DR}}$ from the horizontal reflectivity measurements $\bar{Z}_{h}$ objectively indicates the zones in which the $\bar{Z}_{\mathrm{DR}}$ measurement can be significantly contaminated. This objective assessment of the $\bar{Z}_{\mathrm{DR}}$ measurement quality may improve the qualitative microphysical or the quantitative hydrological analysis of the dual polarization radar data.

This study has shown that the instrumental error of the ratio parameter measurements may partly arise from the mismatch in the main lobe of the illumination functions at each wavelength or polarization. Therefore, the Potter feed horn and the aerial should be optimally designed to reduce this mismatch in the main lobe as well as to decrease below $-30 \mathrm{~dB}$ the relative amplitude of the illumination functions in the sidelobes. In any cases, the illumination functions of the antenna should be measured as accurately as possible for all antenna polar angles $\gamma^{\prime}$ and $\psi^{\prime}$ in order to estimate the antenna induced errors from three-dimensional measurements.

Acknowledgments. The differential reflectivity measurement capability has been implemented on the ANATOL radar under the Direction des Recherches et Etudes Techniques Contract 83-34-173-00-470-75-01, with the technical participation of the Centre National d'Etudes des Télécommunications (C.N.E.T.) for the antenna system. The experiments have been carried out under the Ministère de la Recherche et de la Technologie (M.U.L.T./M.R.T.) Contract 84F 0497. The authors are indebted to all campaign participants, to
R. Pejoux and F. Besserve for their help in the data treatments, to the referees for their comments, and to C. Paquet and J. Squarise for typing and editing this manuscript.

\section{REFERENCES}

Atlas, D., and F. H. Ludlam, 1961: Multi-wavelength radar reflectivity of hailstorms. Quart. J. Roy. Meteor. Soc., 87, 523-534.

Behe, R., and R. Nurdin, 1984: Etude et réalisation d'une source primaire duplexée pour le radar météorologique "ANATOL." Note Technique C.N.E.T.-NT/PAB/ETR/ANT/341.

Bringi, V. N., T. A. Seliga and S. M. Cherry, 1983: Statistical properties of the dual-polarization differential reflectivity $\left(Z_{\mathrm{DR}}\right)$ radar signal. I.E.E.E. Trans. Geosci. Remote Sensing, 21, 215-220.

Cordesses, R., J. Fournet-Fayard, D. Ramond and P. Amayenc, 1983: ANATOL: A S-band differential reflectivity radar for meteorological studies. Preprint 21st Conf. on Radar Meteorology, Edmonton, Amer. Meteor. Soc., 358-361.

Donaldson, R. J., 1964: A demonstration of antenna beam errors in radar reflectivity patterns. J. Appl. Meteor., 3, 611-623.

- 1965: Resolution of a radar antenna for distributed targets. $J$. Appl. Meteor., 4, 727-740.

Herzegh, P. H., and R. E. Carbone, 1984: The influence of antenna illumination function characteristics on differential reflectivity measurements. Preprint 22nd Conf. on Radar Meteorology, Zurich, Amer. Meteor. Soc., 281-286.

- and J. W. Conway, 1986: On the morphology of dual-polarization radar measurements: Distinguishing meteorological effects from radar system effects. Preprint 23rd Conf. on Radar Meteorology, Snowmass, Amer. Meteor. Soc., 3, 55-58.

Jameson, A. R., and A. J. Heymsfield, 1984: Comments on "Antenna beam patterns and dual-wavelength processing." J. Climate Appl. Meteor., 23, 855-858.

Johnson, D. B., 1984: The effect of antenna sidelobes on multipleparameter radar measurements. J. Atmos. Oceanic Technol., 1, 287-290.

Keeler, R. J., P. H. Herzegh and C. L. Frush, 1984: Measurements of the CP-2 copolar and cross-polar antenna illumination functions at S- and X-band. Preprint 22nd Conf. on Radar Meteorology, Zurich, Amer. Meteor. Soc., 287-291.

Rinehart, R. E., and J. D. Tuttle, 1982: Antenna beam patterns and dual-wavelength processing. $J$. Appl. Meteor., 21, 1865-1880 (Corrigendum, 1983, J. Climate Appl. Meteor., 22, 1132).

$\longrightarrow$, and - 1984a: Dual-wavelength processing: Some effects of mismatched antenna beam patterns. Radio Sci., 19, 123-131.

$\longrightarrow$, and - 1984b: Reply. J. Climate Appl. Meteor., 23, 859861.

Sachidananda, M., and D. S. Zrnić, 1985: $Z_{\mathrm{DR}}$ measurement considerations for a fast scan capability radar. Radio Sci., 20, 907922.

Seliga, T. A., and V. N. Bringi, 1976: Potential use of radar differential reflectivity measurements at orthogonal polarizations for measuring precipitation. J. Appl. Meteor., 15, 69-76.

Zawadski, I., 1982: The quantitative interpretation of weather radar measurements. Atmos. Ocean, 20, 158-180. 